\title{
Oral midazolam reduces cortisol levels during local anaesthesia in children: a randomised controlled trial
}

\author{
Heloisa Sousa GOMES(a) \\ Patrícia CORRÊA-FARIA ${ }^{(b)}$ \\ Tarcília Aparecida SILVA(c) \\ Saul Martins PAIVA ${ }^{(b)}$ \\ Paulo Sérgio Sucasas COSTA ${ }^{(d)}$ \\ Aline Carvalho BATISTA ${ }^{(e)}$ \\ Luciane Rezende $\operatorname{COSTA}^{(\mathrm{f})}$ \\ (a) Universidade Federal de Goiás - UFG, \\ Programa de Pós-Graduação em \\ Odontologia, Goiânia, GO, Brazil. \\ (b) Universidade Federal de Minas \\ Gerais - UFMG, Faculdade de Odontologia, \\ Departamento de Odontopediatria e \\ Ortodontia, Belo Horizonte, MG, Brazil. \\ (c) Universidade Federal de Minas Gerais \\ - UFMG, Faculdade de Odontologia, \\ Departamento de Cirurgia Bucal e Patologia, \\ Belo Horizonte, MG, Brazil. \\ (d) Universidade Federal de Goiás - UFG, \\ Faculdade de Medicina, Departamento de \\ Pediatria, Goiânia, GO, Brazil. \\ (e) Universidade Federal de Goiás - UFG, \\ Faculdade de Odontologia, Departamento \\ de Ciências Estomatológicas (Patologia \\ Bucal), Goiânia, GO, Brazil. \\ (f) Universidade Federal de Goiás - UFG, \\ Faculdade de Odontologia, Goiânia, GO, Brazil.
}

Declaration of Interests: The authors certify that they have no commercial or associative interest that represents a conflict of interest in connection with the manuscript.

Corresponding Author:

Aline Carvalho Batista

E-mail:ali.caba@uol.com.br

DOI: 10.1590/1807-3107BOR-2015.vol29.0125

Submitted: Nov 14, 2014

Accepted for publication: Jun 19, 2015

Last revision: Aug 26, 2015

\begin{abstract}
Little is known about whether midazolam sedation can reduce salivary cortisol levels and consequently influence children's behaviour during dental treatment. The aim of this study was to evaluate the effect of midazolam sedation on salivary cortisol and its correlation with children's behaviour during restorative dental treatment. Eighteen healthy children, aged two to five years, were randomly assigned to two dental treatment appointments, both with physical restraint: oral midazolam $1 \mathrm{mg} / \mathrm{kg}$ (MS) and placebo (PS). An observer assessed the children's behaviour (videos) using the Ohio State University Behavioral Rating Scale (OSUBRS). The children's saliva was collected just after waking up, on arrival at the dental school, 25 minutes after local anaesthesia, and 25 minutes after the end of the procedure. Salivary cortisol levels were determined using the enzyme-linked immunoabsorbent assay. The data were analysed by bivariate tests and multivariate analysis of variance ( $5 \%$ level). Salivary cortisol levels were lower in the MS group than in the PS group at the time of anaesthesia $(p=0.004)$, but did not vary during the appointment within sedation $(p=0.319)$ or placebo $(p=0.080)$ groups. Children's behaviour was negative most of the time and did not differ between MS and PS; however, the behaviour (OSUBRS) did not correlate with salivary cortisol levels. Oral midazolam is able to control salivary cortisol levels during dental treatment of pre-schoolers, which might not lead to better clinical behaviour.
\end{abstract}

Keywords: Saliva; Hydrocortisone; Midazolam; Child Behaviour; Dental Anxiety.

\section{Introduction}

One of the most difficult problems during dental treatment of pre-schoolers is behaviour management. ${ }^{1}$ Local anaesthesia is the most commonly cited cause of anxiety and stress in children during dental treatment. ${ }^{2}$ Moreover, factors such as youth, previous negative experiences, anxiety, and toothache have contributed to children's negative behaviour in the dental school. ${ }^{3}$

Many techniques have been used to control children's behaviour during dental treatment. The basic methods are not satisfactory for all children, making it necessary to use advanced methods. These include sedation techniques (minimal, moderate, and deep), physical restraint, 
and general anaesthesia. ${ }^{4}$ Moderate sedation with oral midazolam is a pharmacological method used in dentistry when basic behavioural techniques do not allow adequate dental treatment. ${ }^{5}$ However, a systematic review has concluded that the evidence for midazolam as an effective sedative in behavioural control of children up to 16 years of age undergoing dental treatment is weak, and that clinical trials demonstrating its effectiveness are lacking. ${ }^{6}$

Assessments of the effectiveness of sedatives in paediatric dentistry have relied on behavioural scales. In addition to these scales, there are physical (e.g., blood pressure and oxygen saturation) and biochemical methods (e.g., plasma or salivary cortisol levels). Cortisol levels in the saliva of children under dental treatment are a biochemical marker that is associated with anxiety. ${ }^{7,8}$ This method offers advantages such as ease of collection, noninvasiveness, safety, and painlessness. ${ }^{9}$ Reduced stress and anxiety are reflected in children's salivary cortisol levels. ${ }^{8,10}$ This result has clinical implications, since less stress may lead to better child behaviour during dental treatment. ${ }^{3}$

In stressful situations such as local anaesthesia administration, ${ }^{2}$ two systems, the sympathetic nervous system (SNS) and the hypothalamic-pituitary-adrenal (HPA) axis, interact. ${ }^{11}$ Activation of the HPA axis results in an increase in the secretion of cortisol into the bloodstream, slowing down the response to a stress stimulus. ${ }^{11,12}$ Thus, cortisol in saliva increases gradually until reaching a peak 20 to 30 minutes after the stress stimulus. ${ }^{13}$

The literature (PubMed database, search terms: oral midazolam, dental anaesthesia, child behaviour, and salivary cortisol) provides no evidence of an association between dental anxiety and pre-schoolers' behaviour during dental treatment under sedation. On the basis of a randomised controlled triple-blind crossover clinical trial, this study assessed: 1) the salivary cortisol levels of 2-5-year-olds sedated with oral midazolam during local anaesthesia for restorative dental treatment; and 2) the relationship between salivary cortisol and behaviour measured on an observational scale. The hypothesis was that midazolam would reduce stress and improve behaviour during dental treatment of under-sixes.

\section{Methodology Study design}

This randomised, controlled, crossover, and triple-blind clinical trial was approved by the Institutional Research Ethics Board (\#307/2011) and registered in the Clinical Trials Database (NCT01795222). A placebo control group was used to assess children's behaviour in comparison with the same child's treatment under sedation. Parents were told that a placebo would be used in one of the sessions and were asked to sign an informed consent form after being informed of the objectives, procedures, risks, and benefits of the study.

\section{Participants}

This study included children who were referred to a dental sedation centre between April 2012 and December 2012. The sedation protocols followed established guidelines. ${ }^{4}$ Healthy children aged 2 to 5 years, American Society of Anesthesiologists (ASA) I or II, requiring at least two restorative procedures and who had displayed negative behaviour during earlier dental appointments (definitely negative or negative according to the Frankl Behavior Rating Scale $)^{14}$ were included. Exclusion criteria were previous dental treatment under sedation and recent use of systemic corticosteroids. ${ }^{11}$

Sample size was calculated on the basis of a pilot study with eight children. In the pilot study, salivary cortisol levels 25 minutes after anaesthesia had a mean (standard deviation) of $0.33(0.25) \mu \mathrm{g} / \mathrm{dL}$ in the midazolam group and $0.88(0.64) \mu \mathrm{g} / \mathrm{dL}$ in the placebo group. Thus, a sample of 14 patients was needed for this crossover study of two groups to have an $81 \%$ probability of detecting a difference between treatments at a 5\% significance level if the real difference between the treatments was 0.60 .

\section{Dental examination}

An integrated medical-dental examination was carried out during the first visit to determine eligibility for the study. Oral examination began with prophylaxis with pumice in a rubber cup at low speed and with flossing. Oral condition and consequent dental need were assessed using the decayed, missing, or filled primary teeth $(\mathrm{dmft})$ index.${ }^{15}$ No sedation techniques 
were used in the first visit; children who did not cooperate were stabilised by one parent, who was present during the entire procedure and sat in the dental chair with the child.

\section{Intervention groups}

The analysis groups for this study were: MS - oral midazolam syrup (Dormire ${ }^{\circledR} 2 \mathrm{mg} / \mathrm{mL}$, Cristalia, Brazil) at a dose of $1.0 \mathrm{mg} / \mathrm{kg}$ (maximum dose $20 \mathrm{mg}$ ); PS - oral placebo syrup (same characteristics as those of the active drug, magistral preparation). Syrups were administered with a syringe so that a child would receive the same volume in each session. Children in both groups received passive physical restraint (a sheet stabilised with tape) during the two sessions.

One member of the team carried out a permuted-block randomisation of participants (http://www.stattools.net/index.php). Only the paediatrician and anaesthesiologist knew what substance would be administered in the event of an emergency. Thus, the principal investigator and dental staff were masked to avoid any observer influence on the diagnosis during subsequent evaluation of a child's behaviour. Parents were also masked to avoid any influence on the child's behaviour.

\section{Dental treatment sessions}

Each child's sessions were scheduled at the same time in the morning (from 8 to 10 a.m.) at 7-day intervals. Child and parent were sent to a medication administration room, where the anaesthesiologist administered the randomised substance orally. After administration, the child was monitored for 15 minutes and then taken to the dental chair, wrapped in a sheet stabilised with tape, and dental treatment was initiated 20 minutes after the medication had been administered.

The child was seen by the same paediatric dentist in both sessions and was not separated from his or her parent at any time. In each session, only one tooth was restored under local anaesthesia ( $2 \%$ lidocaine with epinephrine 1:100,000, Nova DFL, Rio de Janeiro, Brazil) and rubber dam isolation. The anaesthetic techniques used were inferior alveolar nerve block or maxillary infiltration according to the child's dental treatment needs in each session. A mouth prop was used as needed. The child's blood pressure, respiratory rate (observation of thoracic-abdominal movements), heart rate, and oxygen saturation were continuously monitored during the intervention using a pulse oximeter (Datex-Ohmeda, Helsinki, Finland).

At the end of each appointment, parents were given written postoperative recommendations. Discharge followed strict criteria ${ }^{16}$ On the following day, the parent was contacted to inform about possible adverse events.

\section{Salivary cortisol assessment}

The saliva was collected with gloved hands using Salivette ${ }^{\circledast}$ tubes (Sarstedt Inc., Nümbrecht, Germany). First, the researcher in charge took the first saliva sample when the child woke up at home, on the day prior to the dental appointment. The other four samples were taken during each day of clinical care. One sample was taken by the parent when the child woke up at home and the others were taken by the researcher upon arrival at the dental school, 25 minutes after anaesthesia, and 25 minutes after the procedure.

After collection, the Salivette ${ }^{\circledR}$ tubes were centrifuged at $3000 \mathrm{rpm}$ for 15 minutes (Sislab/Basic, São Paulo, Brazil). They were subsequently stored in Eppendorf tubes and frozen at $-80^{\circ} \mathrm{C}$ (Sanyo/ Vip ${ }^{\circledR}$ Plus ${ }^{\mathrm{TM}}$, Wood Dale, USA) until the time of analysis. An enzyme immunoassay kit (Salimetrics, State College, USA) was used to measure cortisol concentration in the saliva samples. The samples were evaluated using a microplate reader (Molecular Devices, Spectra Max 190, Sunnyvale, USA) at 450 $\mathrm{nm}$ absorbance. Cortisol levels were determined using the standard curves prepared according to the manufacturer's instructions. The detection limits ranged from $0.012 \mu \mathrm{g} / \mathrm{dL}$ to $3000 \mu \mathrm{g} / \mathrm{dL}$.

\section{Child behaviour assessment}

Every clinical session during the two appointments of clinical treatment was filmed for subsequent behavioural analysis. The children's behaviour was assessed by a previously trained and masked observer using the Ohio State University Behavioral Rating Scale (OSUBRS). ${ }^{17} \mathrm{~A}$ gold-standard paediatric dentistry researcher watched 
videos showing treatment of children outside the present study, in order to evaluate agreement between the researcher and the observer. Intra- and inter-observer agreement was measured at 7-day intervals and had Kappa values of 0.9 and 0.8 , respectively.

The OSUBRS is simple and easily performed. It consists of four points involving head and limb movements, crying, and physical resistance: 1) no crying and no movement (quiet); 2) crying and no movement; 3) movement without crying; and 4) crying and movement (struggling). For this study, OSUBRS scoring involved a slight modification of the original scale. The behaviour of all the children was evaluated minute by minute to obtain the mean of the percentage of each score in a given treatment session and at the following specific times: during local anaesthesia and at the end of the whole procedure.

\section{Statistical analyses}

Data were entered and analysed using the IBM SPSS 22.0 (IBM Corporation, New York, USA) and Prism software (GraphPad Prism 6; GraphPad Software, La Jolla, USA). The significance level for statistical tests was set at $5 \%$.

Salivary cortisol values for each patient at different times (upon waking before the session, upon arrival at the dental school, 25 minutes after anaesthesia, and 25 minutes after the procedure) were transformed into area under the curve (AUC). ${ }^{18}$

The groups, MS and PS, were compared regarding salivary cortisol and OSUBRS scores through repeated measures tests: Wilcoxon for non-parametric data and paired Student's t-test for parametric data. Variations in salivary cortisol levels in the same group, assessed at different times, were tested by multivariate analysis of variance (MANOVA), Pillai's trace criterion. The correlation between salivary cortisol and OSUBRS was tested by Spearman's (non-parametric data) or Pearson (parametric data) analyses.

\section{Results}

Thirty-one children were examined for inclusion in this study (Figure 1). The 18 children included in the study (61.1\% girls) were 46.8 (standard deviation "SD" 13.9) months old, weighed 15.9 (SD 3.4) kg, and had a dmft index of 5.5 (SD 2.7).
The planned treatments for the two sessions were concluded in all cases. During treatment, the children in the MS and PS groups respectively received class I ( $33.3 \%$ vs. $30.5 \%)$, class II ( $2.7 \%$ vs. $5.5 \%)$, and class III restorations (13.8\% vs. $13.8 \%$ ). The anaesthetic techniques used in the MS and PS groups were inferior alveolar nerve block (38.9\% vs. $44.5 \%$, respectively) and maxillary infiltration (61.1\% vs. 55.5\%, respectively) (Fisher's exact test $p=0.145)$. The type of local anaesthesia technique varied according to the child's need - 13 children received the same technique in both sessions, whilst 5 received different techniques in each session. Treatment sessions lasted 28.6 (SD 6.5) minutes (MS) and 33.3 (SD 7.7) minutes (PS) $(p=0.021)$.

Salivary cortisol levels varied during the evaluations of dental treatment with and without sedation (Table 1 and Figure 2), but had a normal distribution (Kolmogorov-Smirnov test $\mathrm{p}>0.05$ ). The paired comparison of salivary cortisol levels in children in both sessions (MS and PS) showed that, at the time of anaesthesia, cortisol levels were lower when the child received midazolam than when s/he received placebo (Table 1 and Figure 2). The salivary levels related to the two anaesthetic techniques did not differ in the MS ( $p=0.392$, Mann-Whitney test) or PS ( $p=0.724)$ groups. There was no statistically significant difference in cortisol levels between MS and PS at the other saliva collection times ( $p=0.132$ - upon waking, on the day of the session; $p=0.441$ - on arrival at the dental school; $\mathrm{p}=0.494-25$ minutes after the end of the procedure). There was greater variation in cortisol levels in the same session when children received placebo $(p=0.080)$ than when they received midazolam $(p=0.319)$, but this was not statistically significant (MANOVA, Pillai's trace criterion). There was no statistically significant difference between the first and second sessions in a paired evaluation of salivary cortisol levels on arrival at the dental school $(p=0.261)$.

After 20 minutes of drug administration, at the beginning of the dental treatment, children in the MS group were drowsy but responsive. The observed behaviour varied widely (Table 2), with an OSUBRS score of 4 (struggling) during local anaesthesia predominating in both the MS and PS groups. The MS group had a higher percentage of score 3 


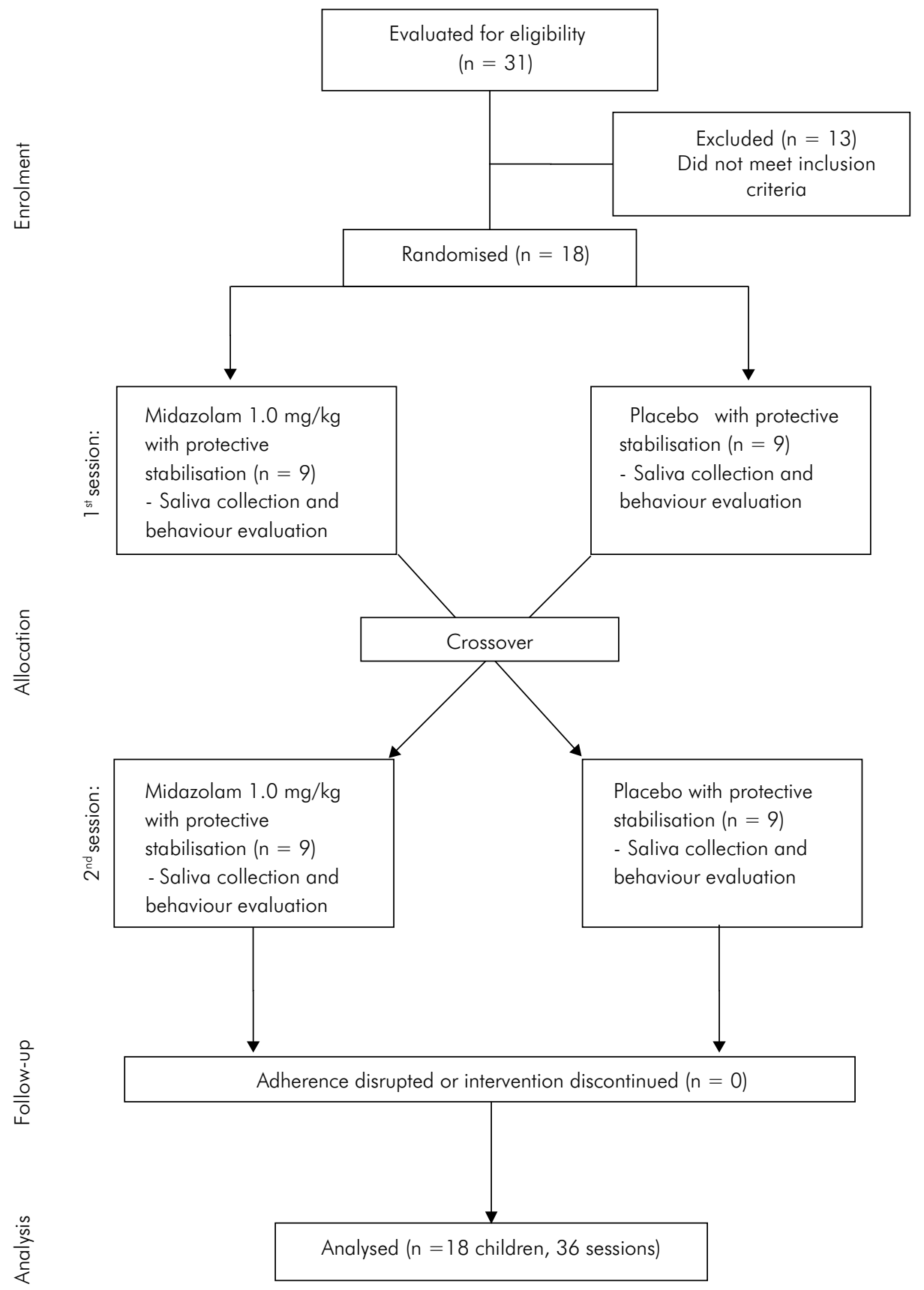

Figure 1. Consolidated Standards of Reporting Trials (CONSORT) statement flow diagram.

(movement without crying) during anaesthesia. After treatment, the behaviour of children in the two groups was similar. There was no statistically significant correlation between salivary cortisol levels and struggling (percentage of score 4) during local anaesthesia, at the end of dental treatment, or throughout the session for either of the groups (Table 3).
Heart rate and oxygen saturation remained within the normal range during the use of both the sedative and the placebo. There were no adverse intraoperative events in either group. In the first 24 postoperative hours, two children from the MS group had nausea and vomiting and one had excessive sleepiness. 
Table 1. Salivary cortisol levels during dental treatment in children under sedation with midazolam (MS) or placebo (PS).

\begin{tabular}{|c|c|c|c|}
\hline \multirow{2}{*}{$\begin{array}{l}\text { Salivary cortisol level }(\mu \mathrm{g} / \mathrm{dL}) \\
\text { Variables }\end{array}$} & \multicolumn{2}{|c|}{ Mean (standard deviation) or median (minimum-maximum) } & \multirow{2}{*}{$\mathrm{p}$-value } \\
\hline & $M S(n=18)$ & $\mathrm{PS}(\mathrm{n}=18)$ & \\
\hline \multicolumn{4}{|l|}{ Time of measurement, mean (standard deviation) } \\
\hline Upon waking at home & \multicolumn{2}{|c|}{$0.33(0.12)$} & \\
\hline Upon waking on the day of the session* & $0.32(0.32)$ & $0.34(0.18)$ & 0.132 \\
\hline On arrival at the dental school* & $0.25(0.12)$ & $0.27(0.09)$ & 0.441 \\
\hline 25 minutes after anaesthesia* & $0.47(0.42)$ & $1.05(0.64)$ & $0.004^{\#}$ \\
\hline 25 minutes after the end of the procedure* & $0.80(0.90)$ & $0.93(0.69)$ & 0.494 \\
\hline Area under the curve* & $1.04(0.88)$ & $1.57(0.94)$ & 0.054 \\
\hline \multicolumn{4}{|c|}{ Anaesthetic technique, median (minimum-maximum) } \\
\hline Inferior alveolar nerve block ${ }^{+}$ & $1.42(0.11-1.58)$ & $0.60(0.30-2.35)$ & 0.655 \\
\hline Maxillary infiltration ${ }^{+}$ & $0.27(0.12-1.01)$ & $0.82(0.45-2.06)$ & $0.012^{\#}$ \\
\hline
\end{tabular}

* Paired Student's t-test (mean and standard deviation).

+ Wilcoxon test (median and minimum-maximum values).

* Statistically significant.

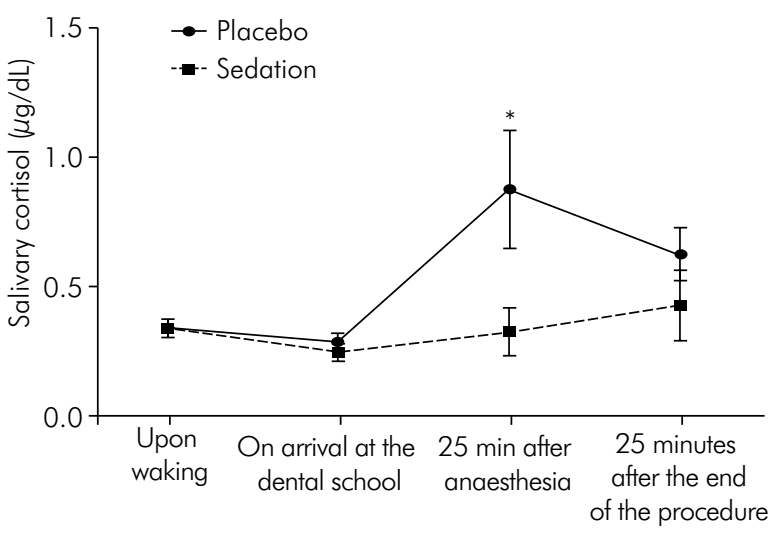

*statistically significant $(\mathrm{p}=0.004)$.

Figure 2. Comparison of patients' salivary cortisol levels (mean and standard deviation, in $\mu \mathrm{g} / \mathrm{dL}$ ) during dental treatment with sedation (MS) and placebo (PS) at different collection times. Paired Student's t-test.

\section{Discussion}

The key finding of this study was that children presenting with uncooperative behaviour during dental sedation with midazolam might not be stressed. Accordingly, oral midazolam at a dose of $1.0 \mathrm{mg} / \mathrm{kg}$ was able to reduce pre-schoolers' salivary cortisol, a biomarker of stress, during restorative dental treatment, mainly during local anaesthesia administration. However, this reduction in salivary cortisol levels did not impact on better children's behaviour during stressful dental treatment situations. Several factors may have influenced these findings and will be discussed further ahead.
Several studies have shown an increase in salivary cortisol levels during the most stressful dental treatment procedures ${ }^{10,19,20,21}$ as well as during intravenous access in medical procedures. ${ }^{22}$ However, these studies were conducted with unsedated patients. ${ }^{78,9,10,19,20}$ In the cortisol assessments "upon waking" and "upon arrival at the dental school", there were no statistically significant differences between the MS and PS groups. There was no stressful stimulus prior to drug administration, and salivary cortisol levels did not differ significantly between the group receiving the active drug and the one that received the placebo, thus confirming other study findings. ${ }^{21}$ Besides, there was no difference in salivary cortisol levels at the end of the procedure, because cortisol decreased in the PS group children after they received local anaesthesia. Accordingly, aversive stimuli after anaesthesia - motor noise and vibration, mouth prop, and rubber dam isolation - would not cause cortisol changes even in children under the placebo effect. This should be further investigated in future studies.

Behaviour management problems during paediatric dental treatment is not necessarily associated with dental anxiety, ${ }^{1}$ which means that a child might be uncooperative with a dental procedure without having dental anxiety. This study supports this knowledge, as the subjective measure (OSUBRS) was related to child's behaviour and the objective assessment (salivary cortisol) to child's anxiety. Midazolam was associated with lower release of cortisol in saliva 
Table 2. Children's behaviour during dental treatment under midazolam sedation (MS) or placebo (PS).

\begin{tabular}{|c|c|c|c|}
\hline \multirow{2}{*}{ Behavioural variables } & \multicolumn{2}{|c|}{ Mean (standard deviation) or median (minimum-maximum) } & \multirow{2}{*}{$\mathrm{p}$-value } \\
\hline & $M S(n=18)$ & $\operatorname{PS}(n=18)$ & \\
\hline \multicolumn{4}{|l|}{ Percentage of " 1 " scores - quiet } \\
\hline During anaesthesia ${ }^{+}$ & $0(0-50.0)$ & $0(0-100)$ & 0.713 \\
\hline Immediately after the end of procedure ${ }^{+}$ & $0(0-100)$ & $0(0-100)$ & 0.655 \\
\hline During the entire treatment session* & $18.3(22.3)$ & $18.6(20.4)$ & 0.968 \\
\hline \multicolumn{4}{|c|}{ Percentage of "2" scores - crying without movement } \\
\hline During anaesthesia + & $0(0-66.7)$ & $0(0-100)$ & 0.210 \\
\hline Immediately after the end of procedure ${ }^{+}$ & $0(0-100)$ & $0(0-100)$ & 0.414 \\
\hline During the entire treatment session* & $20.8(25.5)$ & $27.8(31.9)$ & 0.280 \\
\hline \multicolumn{4}{|c|}{ Percentage of " 3 " scores - movement without crying } \\
\hline During anaesthesia ${ }^{+}$ & $0(0-100)$ & 0 & 0.041 \\
\hline Immediately after the end of procedure ${ }^{+}$ & 0 & $0(0-100)$ & 0.157 \\
\hline During the entire treatment session* & $8.6(12.1)$ & $4.8(11.5)$ & 0.154 \\
\hline \multicolumn{4}{|l|}{ Percentage of " 4 " scores - struggling } \\
\hline During anaesthesia $^{+}$ & $58.4(0-100)$ & $100(0-100)$ & 0.589 \\
\hline Immediately after the end of procedure ${ }^{+}$ & $0(0-100)$ & $0(0-100)$ & 0.180 \\
\hline During the entire treatment session* & $52.6(29.7)$ & $48.8(33.3)$ & 0.516 \\
\hline \multicolumn{4}{|l|}{ Scores as per anaesthetic technique } \\
\hline Inferior alveolar nerve block ${ }^{+}$ & $4.0(1.0-4.0)$ & $4.0(1.0-4.0)$ & 0.317 \\
\hline Maxillary infiltration ${ }^{+}$ & $4.0(2.0-4.0)$ & $4.0(2.0-4.0)$ & 0.414 \\
\hline
\end{tabular}

* Paired Student's t-test (mean and standard deviation).

+ Wilcoxon test (median and minimum-maximum values).

Table 3. Correlation between salivary cortisol levels and struggling behaviour during dental treatment of children under midazolam sedation (MS) or placebo (PS).

\begin{tabular}{lcc}
\hline & MS & PS \\
\hline During anaesthesia & Rho $=0.17$ & Rho $=0.33$ \\
End of procedure & $p=0.55^{*}$ & $p=0.22^{*}$ \\
& Rho $=0.24$ & Rho $=0$ \\
& $p=0.41^{*}$ & $p=1.0^{*}$ \\
\hline
\end{tabular}

* Spearman's correlation test.

compared with placebo during local anaesthetic administration; possibly, children's anxiety was effectively reduced by the sedative during this stressful procedure, but the children's age range did not allow us to assess their anxiety with a validated psychometric measure. Although there was no control of the group to which a child would be assigned in the first or second session, another study has shown that the type of anaesthetic technique used does not influence the next treatment session; ${ }^{23}$ this should be further investigated.

However, the lack of correlation between the biochemical measurement (cortisol) and the clinical measurement (behaviour) is intriguing. Other studies also showed no association between salivary cortisol levels and scores on other behavioural scales in unsedated adults during emergency treatment ${ }^{19}$ and adults sedated with sublingual midazolam during surgical treatment of third molars. ${ }^{21}$

Children's behaviour can be influenced by a number of factors such as temperament, child's age, cognitive development, and social status. ${ }^{1}$ Moreover, compared to adults, children and the elderly might show unexpected behaviour such as agitation and arousal during benzodiazepine sedation. ${ }^{24}$ Thus, although cortisol levels may increase in a stressful situation such as local anaesthesia, moderate sedation still allows children to perceive their environment and express resistance to the procedure. In the present study, for example, children in the MS group had more movement without crying than those in the PS group, i.e., children wanted to avoid the procedure even though they did not cry.

This study had a crossover design in which a child was his/her own control, which has the advantage of minimising other emotional and cognitive biases 
associated with behaviour during dental treatment. Although a washout time of 7 days was established in this and previous crossover studies, ${ }^{25}$ we cannot rule out the possibility of a positive or negative result in the first session influencing the outcome of the second session. However, it can be assumed that midazolam-induced anterograde amnesia would minimize the memory of a positive or negative experience at the dentist's. Furthermore, this study did not show any influence of first-session salivary cortisol levels upon arrival at the dental school on levels during the second session.

This trial has other strengths: it was randomised to prevent observer influence and masked so that the dental team, the parent, and the observer did not know which substance was administered; dental treatment was standardised, as just one tooth at a time was restored under local anaesthesia and in rubber dam isolation.

However, it is important to note that the sample size was calculated with partial results at the time of anaesthesia, perhaps the most critical time in terms of

\section{References}

1. Klingberg G, Broberg AG. Dental fear/anxiety and dental behaviour management problems in children and adolescents: a review of prevalence and concomitant psychological factors. Int J Paediatr Dent. 2007;17(6):391-406. doi:10.1111/j.1365-263X.2007.00872.x

2. Alsarheed M. Children's perception of their dentists. Eur J Dent. 2011;5(2):186-90.

3. Xia B, Wang CL, Ge LH. Factors associated with dental behaviour management problems in children aged 2-8 years in Beijing, China. Int J Paediatr Dent. 2011;21(3):200-9. doi:10.1111/j.1365-263X.2011.01111.x

4. American Academy on Pediatric Dentistry. Clinical Affairs Committee-Behavior. Management Subcommittee. Guideline on behavior guidance for the pediatric dental patient. Pediatr Dent. (2013-2014);35:176-87.

5. Lima AR, Costa LR, Costa PS. A randomized, controlled, crossover trial of oral midazolam and hydroxyzine for pediatric dental sedation. Pesqui Odontol Bras. 2003;17(3):206-11. doi:10.1590/S1517-74912003000300002

6. Lourenço-Matharu L, Ashley PF, Furness S. Sedation of children undergoing dental treatment. Cochrane Database Syst Rev. 2012;(3):CD003877. doi:10.1002/14651858.CD003877.pub4 increased cortisol release due to pain stimulation. For this reason, other differences between the compared variables may not have been demonstrated due to sample size. To make up for this limitation in the present study, it would be worthwhile to conduct additional studies with larger samples to also compare other sedatives that might be more effective for this age group.

\section{Conclusion}

Oral midazolam may help to reduce pre-schoolers' salivary cortisol levels during dental treatment, and especially during local anaesthesia. Regarding children's behaviour, its clinical efficacy in that age group is questionable.

\section{Acknowledgments}

We would like to thank the Conselho Nacional de Desenvolvimento Científico e Tecnológico (CNPq), and the Coordenação de Aperfeiçoamento de Pessoal de Nível Superior (CAPES) for partially funding this study.

7. Hellhammer DH, Wüst S, Kudielka BM. Salivary cortisol as a biomarker in stress research. Psychoneuroendocrinology. 2009;34(2):163-71. doi:10.1016/j.psyneuen.2008.10.026

8. Yfanti K, Kitraki E, Emmanouil D, Pandis N, Papagiannoulis L. Psychometric and biohormonal indices of dental anxiety in children: a prospective cohort study. Stress. 2014;17(4):296-304. doi:10.3109/10253890.2014.918602

9. Golden SH, Wand GS, Malhotra S, Kamel I, Horton K. Reliability of hypothalamic-pituitary-adrenal axis assessment methods for use in population-based studies. Eur J Epidemiol. 2011;26(7):511-25. doi:10.1007/s10654-011-9585-2

10. Kambalimath HV, Dixit UB, Thyagi PS. Salivary cortisol response to psychological stress in children with early childhood caries. Indian J Dent Res. 2010;21(2):231-7. doi:10.4103/0970-9290.66642

11. Hanrahan K, McCarthy AM, Kleiber C, Lutgendorf S, Tsalikian E. Strategies for salivary cortisol collection and analysis in research with children. Appl Nurs Res. 2006;19(2):95-101. doi:10.1016/j.apnr.2006.02.001

12. Jessop DS, Turner-Cobb JM. Measurement and meaning of salivary cortisol: a focus on health and disease in children. Stress. 2008;11(1):1-14. doi:10.1080/10253890701365527 
13. Ali N, Pruessner JC. The salivary alpha amylase over cortisol ratio as a marker to assess dysregulations of the stress systems. Physiol Behav. 2012;106(1):65-72. doi:10.1016/j.physbeh.2011.10.003

14. Frankl SN, Shiere FR, Fogels HR. Should the parent remain with the child in the dental operatory. J Dent Child. 1962;29:150-63.

15. World Health Organization. Oral health surveys, basic methods. 4th ed. Geneva: World Health Organization; 1997.

16. American Academy of Pediatrics; American Academy on Pediatric Dentistry. Guideline for monitoring and management of pediatric patients during and after sedation for diagnostic and therapeutic procedures. Pediatr Dent. 2013-4;35:205-21.

17. Lochary ME, Wilson S, Griffen AL, Coury DL. Temperament as a predictor of behavior for conscious sedation in dentistry. Pediatr Dent. 1993;15(5):348-52.

18. Pruessner JC, Kirschbaum C, Meinlschmid G, Hellhammer $\mathrm{DH}$. Two formulas for computation of the area under the curve represent measures of total hormone concentration versus time-dependent change. Psychoneuroendocrinology. 2003;28(7):916-31. doi:10.1016/S0306-4530(02)00108-7

19. Kanegane K, Penha SS, Munhoz CD, Rocha RG. Dental anxiety and salivary cortisol levels before urgent dental care. J Oral Sci. 2009;51(4):515-20. doi:10.2334/josnusd.51.515
20. Hsu AA, Elten K, Chan D, Flynn T, Walker K, Barnhill $\mathrm{J}$ et al. Characterization of the cortisol stress response to sedation and anesthesia in children. J Clin Endocrinol Metab. 2012 Oct;97(10):E1830-5. doi:10.1210/jc.2012-1499

21. Jerjes W, Jerjes WK, Swinson B, Kumar S, Leeson R, Wood PJ et al. Midazolam in the reduction of surgical stress: a randomized clinical trial. Oral Surg Oral Med Oral Pathol Oral Radiol Endod. 2005;100(5):564-70. doi:10.1016/j.tripleo.2005.02.087

22. Ekbom K, Kalman S, Jakobsson J, Marcus C. Effects of midazolam and nitrous oxide on endocrine and metabolic measurements in children. Horm Res Paediatr. 2012;77(5):309-9. doi:10.1159/000337536

23. Ram D, Amir E, Keren R, Shapira J, Davidovich E. Mandibular block or maxillary infiltration: does it influence children's opposition to a subsequent dental visit? J Clin Pediatr Dent. 2012;36(3):245-9. doi:10.17796/jcpd.36.3.n2×8m38866487020

24. Smith DE, Wesson DR. The benzodiazepines: current standards for medical practice. In: Wesson DR, Camber $\mathrm{S}$, editors. Acute and chronic toxicity of benzodiazepines. England: MTP; 1985. p. 227-31. doi:10.1007/978-94-009-4886-0

25. Ribeiro CC, Lula EC, Azevedo IM, Maia MF, Lopes FF. Salivary retention after application of fluoride gel using toothbrush or tray: a crossover trial. Braz Oral Res. 2012;26(6):493-7. doi:10.1590/S1806-83242012000600002 